\title{
The Immediate Reproducibility of T Wave Alternans During Bicycle Exercise
}

\author{
DANIEL M. BLOOMFIELD, * BROOKE S. RITVO,* MICHAEL K. PARIDES,† and \\ MICHAEL H. KIM‡ \\ From the * Division of Cardiology, Department of Medicine, the +Department of Biostatistics, \\ School of Public Health, Columbia University, New York, New York, and the ₹Cardiovascular \\ Division, University of Michigan Health System, Ann Arbor, Michigan
}

BLOOMFIELD, D.M., ET AL.: The Immediate Reproducibility of T Wave Alternans During Bicycle Exercise. A number of recent studies have demonstrated that the microvolt level $T$ wave alternans measured during bicycle exercise can identify patients at high risk for developing malignant ventricular arrhythmias. However, little is known about the reproducibility of $T$ wave alternans measured during bicycle exercise. The purpose of this study was to prospectively evaluate the immediate reproducibility of $T$ wave alternans measured during bicycle exercise testing. Thirty-five patients with congestive heart failure underwent two sequential bicycle exercise tests with the measurement of $T$ wave alternans. The $T$ wave alternans tests were randomly sorted and then classified by two readers who were blinded to the patient and the sequence of the two tests. Tests were classified as determinate (positive or negative) or indeterminate according to previously published criteria. Of the 22 patients that had two determinate $T$ wave alternans tests, $18(82 \%)$ of 22 patients had concordant test results (kappa 0.58). Of the four patients who had discordant test results on the two tests, three patients had one test that was borderline and difficult to interpret. One patient had two sequential tests that were clearly different. Of the ten patients whose initial test was indeterminate, five became determinate on the second test. $T$ wave alternans measured during bicycle exercise has an acceptable reproducibility when measurements are made from two sequential exercise tests performed within a short period. This data suggests that the measurement of $T$ wave alternans during exercise is reliable. Repeating the exercise test with a second measure of $T$ wave alternans during the same session can significantly reduce the proportion of patients with indeterminate test results. (PACE 2002; 25:1185-1191)

\section{T Wave alternans, exercise testing, sudden cardiac death}

\section{Introduction}

$\mathrm{T}$ wave alternans, a 2:1 pattern of alternation in the morphology of the $\mathrm{T}$ wave, has been shown to be a marker of an increased risk for the ventricular tachyarrhythmias that can cause sudden cardiac death. ${ }^{1-3}$ The development of $\mathrm{T}$ wave alternans is highly dependent on heart rate: $\mathrm{T}$ wave alternans often was not present at rest, but only after the heart rate was increased above $\sim 90$ beats/min. ${ }^{1-4}$ The initial human studies of $\mathrm{T}$ wave alternans used atrial pacing to enable the measurement of $\mathrm{T}$ wave alternans at increased heart

Supported in part by the Doris Duke Charitable Foundation, and was conducted in the Irving Center for Clinical Research supported by grant 5 M01 RR-00645, Division of Research Resources, General Clinical Research Centers Program, National Institutes of Health, Bethesda, Maryland

Address for reprints: Daniel Bloomfield, M.D., College of Physicians and Surgeons PH 3-342, 630 West 168th St., New York, NY 10032. Fax: (212) 305-4825; e-mail: dmb9@columbia.edu

Received March 23, 2001; revised July 26, 2001; accepted September 13, 2001. rates with relatively low noise levels. A number of elegant advances in signal processing and noise reduction made it possible to measure $\mathrm{T}$ wave alternans during exercise. ${ }^{4}$ Hohnloser et al. ${ }^{5}$ demonstrated the equivalence of $\mathrm{T}$ wave alternans measured during bicycle exercise or during atrial pacing. A number of studies have demonstrated an association between $\mathrm{T}$ wave alternans meaured during bicycle exercise and an increased risk of subsequent spontaneous arrhythmic events. ${ }^{6-9}$

Despite the potential value of using microvolt level $\mathrm{T}$ wave alternans to identify patients at high risk for developing malignant ventricular arrhythmias, little is known about the reproducibility of T wave alternans measured during bicycle exercise. Accordingly, the purpose of this study was to prospectively evaluate the immediate reproducibility of $\mathrm{T}$ wave alternans measured during bicycle exercise testing.

\section{Methods}

Thirty-five patients who consented to participate and who were enrolled in two larger studies participated in this substudy. One study was an 
epidemiological study of $\mathrm{T}$ wave alternans in patients with congestive heart failure $(n=31)$, the other study evaluated $\mathrm{T}$ wave alternans in patients referred for electrophysiological testing $(n=4)$. $\beta$ adrenergic blocking agents were held on the day of the study. All patients underwent two sequential submaximal bicycle exercise tests on the same day. Following the first exercise test, patients were allowed to rest until they subjectively returned to their baseline state and their heart rate and blood pressure had returned to baseline values. On average, the two exercise tests were performed 15 minutes apart.

\section{T wave Alternans Testing}

Careful skin preparation including mild abrasion and high resolution electrodes (Micro-V Alternans Sensors, Cambridge Heart Inc., Bedford, MA, USA) were used to minimize noise. The same leads were used for both exercise tests (i.e., the leads were not changed after the first test). $\mathrm{T}$ wave alternans was measured with the CH2000/D8 system (Cambridge Heart Inc.) as previously described during submaximal bicycle exercise to achieve a heart rate of 110 beats $/ \mathrm{min}^{6}{ }^{6} \mathrm{~T}$ wave alternans was prospectively defined as positive when it was sustained with an onset heart rate $\leq$ 110 beats/min with an alternans amplitude $\geq 1.9$ $\mu \mathrm{V}$ and alternans ratio $\geq 3$ in the vector magnitude lead, any orthogonal lead or two consecutive precordial leads. ${ }^{10} \mathrm{~T}$ wave alternans was prospectively defined as negative if the criteria for a positive test were not met and if there was a 1-minute period without significant alternans while the heart rate was $\geq 105$ beats $/ \mathrm{min}$ and the tracing was not obscured by noise or ectopic beats. Otherwise, the $\mathrm{T}$ wave alternans test was considered indeterminate.

$\mathrm{T}$ wave alternans tests were read and interpreted by two independent physician readers who were blinded to the patient's clinical data. Tests were randomly sorted and relabeled to prevent the independent physician readers from knowing the sequence of tests or which two tests were from the same patient. All tests were classified as positive, negative, or indeterminate.

\section{Statistical Analysis}

The primary analysis of the data was designed to determine the reproducibility of sequential $\mathrm{T}$ wave alternans exercise tests. Even though the classification of $\mathrm{T}$ wave alternans test has three outcomes (two determinate outcomes [positive and negative]) and an indeterminate outcome), the most important question in determining the reliability of a test is how reliable are the tests that are interpreted as definitive (i.e., determinate). A high concordance between determi- nate tests (positive or negative) is important if the test results are to be considered definitive. The concordance of indeterminate tests, however, is not informative. The authors expected and hoped that some of the indeterminate tests would not be concordant and would become determinate on repeat testing. Therefore, to assess the reliability of $\mathrm{T}$ wave alternans testing, the agreement between two sequential tests in the subset of patients that had two determinate tests was analyzed. The reproducibility of sequential $\mathrm{T}$ wave alternans tests was described by the observed proportion of agreement (proportion of concordant determinant tests) and by a statistical measure of agreement called the kappa statistic. Kappa is a chance-corrected measure of agreement taking values in the range of zero to one, with higher values indicating better agreement. Values above 0.75 indicate excellent agreement, values between 0.40 and 0.75 indicate moderate agreement, and values below 0.40 indicate poor agreement. ${ }^{11}$ In the subset of patients with two positive tests, the onset heart rate for each of the two tests was compared using a paired $t$-test.

\section{Results}

Thirty-five patients were studied. The clinical characteristics of these patients are described in Table I.

\section{Table I.}

Clinical Characteristics

\begin{tabular}{lc}
\hline Age (years) & $53.0 \pm 11.5$ \\
Male sex & $86 \%$ \\
Ejection fraction (mean \%) & $20.4 \pm 5.4$ \\
Etiology of heart disease & \\
$\quad$ Ischemic & $16(45.7 \%)$ \\
Idiopathic & $15(42.8 \%)$ \\
Valvular & $2(5.7 \%)$ \\
Alcohol related & $2(5.7 \%)$ \\
NYHA & \\
NYHA Class I & $8(22.9 \%)$ \\
NYHA Class II & $17(48.6 \%)$ \\
NYHA Class III & $19(25.7 \%)$ \\
NYHA Class IV & $1(2.9 \%)$ \\
Diabetes & $9(25.7 \%)$ \\
History of VT/VF & $2(5.7 \%)$ \\
Medications & \\
ACE inhibitor/angiotensin II blocker & $30(85.7 \%)$ \\
B-blocker & $16(11.4 \%)$ \\
Amiodarone & $2(5.7 \%)$ \\
\hline
\end{tabular}

NYHA = New York Heart Association; VT = ventricular tachycardia; $\mathrm{VF}=$ ventricular fibrillation; $\mathrm{ACE}=$ angiotensinconverting enzyme. 
Table II.

Outcome of Two Sequential T Wave Alternans Tests

\section{Second Test}

\begin{tabular}{lrcc} 
& Positive & Negative & Indeterminate \\
\hline Initial Test & & & \\
Positive & $\mathbf{1 3}$ & $\mathbf{2}$ & 3 \\
Negative & $\mathbf{2}$ & $\mathbf{5}$ & 0 \\
Indeterminate & 2 & 3 & 5 \\
\hline
\end{tabular}

Numbers with bold typeface identify patients who had determinate test results (positive or negative) on both the initial test and the second test.

\section{Concordance of Sequential T Wave Alternans Tests}

The results of the outcome of the two $\mathrm{T}$ wave alternans tests are described in Table II. The
$\mathrm{T}$ wave alternans test was positive in $18(51 \%)$ of the initial tests. Of the 35 patients, 22 had $\mathrm{T}$ wave alternans tests that were determinate (positive or negative) on the first and second tests. Of these 22 patients, $18(82 \%)$ tests were concordant and 4 were discordant (kappa = 0.58). In the $13 \mathrm{~T}$ wave alternans tests that were positive on the first and second test, the average onset heart rate on the first test was $94.1 \pm 9.5$ beats/min. There was no significant difference between the onset heart rate on the first and second test (mean difference $0.85 \pm 10.1$ beats $/ \mathrm{min}$, $\mathrm{P}=0.8$ ). An example of one patient with concordant results (two positive tests) is illustrated in Figure 1.

\section{Discordant T Wave Alternans Tests}

Four patients had discordant test results on the first and second tests. Clinical characteristics
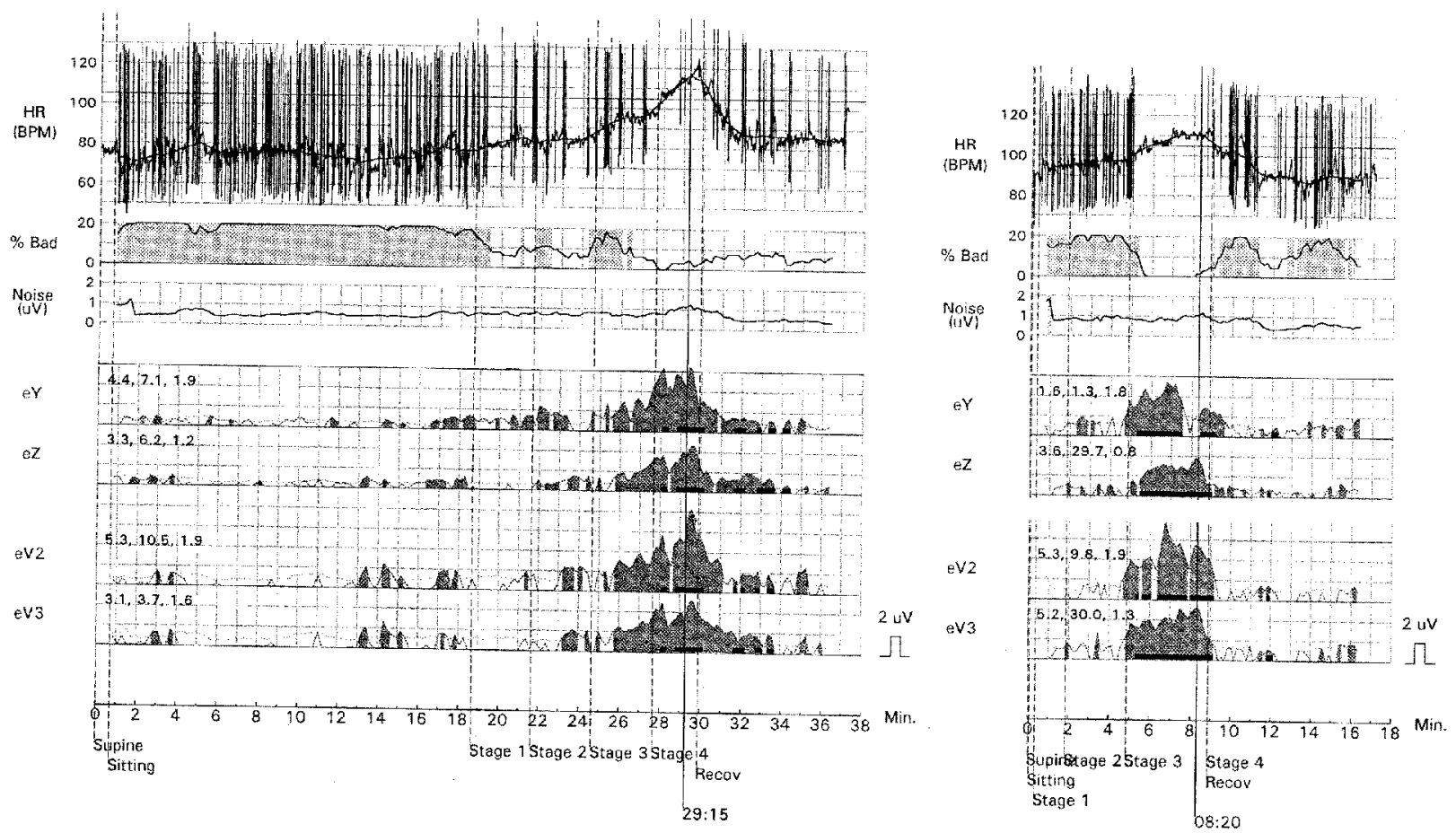

Figure 1. T wave alternans tests are shown for the first test (left panel) and the second test (right panel). In each panel, heart rate is displayed on the upper tracing. The second tracing represents the percentage (\%) of "bad" (i.e., nonsinus) beats and is shaded in grey when $>10 \%$ of the beats in a 128-beat segment are classified as "bad"). The third tracing represents noise levels. T wave alternans is displayed on the lower tracings for the orthogonal leads eY, eZ, and for precordial leads $V_{Z^{-}} V_{3}$. Each small box on the alternans tracings represents $2 \mu V$. The dark black line represents the alternans voltage and the shaded areas indicate that the alternans ratio (K score) is $\geq 3$. In both tests, there is dense ectopy at rest that disappears during exercise. During the first test (top panel) $T$ wave alternans develops at an onset heart rate of 94 beats $/ \mathrm{min}$. During the second test (right panel), $T$ wave alternans develops immediately after the ectopy disappears at an onset heart rate of 99 beats/min. In both tracings, the magnitude of alternans is between 4 and $6 \mu V$ at the maximal heart rate of 110 beats $/ \mathrm{min}$. 
were similar in patients with concordant and discordant results. With regard to drug use, the two patients taking amiodarone had concordant test results. Three of the four patients with discordant test results were taking $\beta$-blockers, in contrast to 13 of the 31 patients with concordant results $(\mathrm{P}=$ 0.2). Of the four patients with discordant results on the first and second tests, one of these four patients had a clear switch from positive to negative (Fig. 2).

In the other three patients with discordant $\mathrm{T}$ wave alternans test tracings, the interpretation of one or both tests was difficult. In two patients, one of the two sequential $\mathrm{T}$ wave alternans tests was extremely difficult to interpret because it was difficult to determine if the alternans that was present was sustained (i.e., consistently present above a threshold heart rate). The $\mathrm{T}$ wave alternans tracings from one of these two patients are illustrated in Figure 3. The final patient with discordant $\mathrm{T}$ wave alternans tests had one test that was difficult to interpret because the $\mathrm{T}$ wave alternans occurred during a period when RR interval alternans was present (Fig. 4). RR interval alternans is a potential cause of artifactual $\mathrm{T}$ wave alternans; $\mathrm{T}$ wave alternans may be the direct result of RR interval alternans rather than a primary abnormality in repolarization.

\section{Effect of Sequential Testing on Indeterminate T Wave Alternans Tests}

Thirteen patients had at least one indeterminate test result. Five (14.3\%) patients had indeterminate test results on both tests and most of these tests were indeterminate because of the patient's inability to achieve the target heart rate of 105 beats/min. Of the 35 initial tests, $10(28.6 \%)$ were indeterminate. The causes of indeterminate test results are described in Table III. Five of these ten tests became determinate on the second test (two positive and three negative).

\section{Discussion}

This study demonstrates that $\mathrm{T}$ wave alternans measured during bicycle exercise has an adequate reproducibility when measurements are made from two sequential exercise tests performed within a short period of time. In the group of patients with determinate test results on both the first and second tests, the results were concordant in $82 \%$ of patients. In addition, among the patients with positive tests, the onset heart rate of the $\mathrm{T}$ wave alternans in the first and second tests were nearly identical. This data suggests that the signal processing and noise reduction algorithms used to measure $\mathrm{T}$ wave alternans during exercise are effective and that the measurement of $\mathrm{T}$ wave alternans during bicycle exercise is reliable.

The data from this study also demonstrated that repeating the measurement of $\mathrm{T}$ wave alternans with a second test done 15 minutes after the conclusion of the first test significantly reduced the rate of indeterminate tests. This suggests that repeating the exercise test with a second measure of $\mathrm{T}$ wave alternans during the same session can significantly reduce the proportion of patients with indeterminate test results. With this approach, only $5(14 \%)$ of 35 had indeterminate test results on both tests and most of these were indeterminate because of inadequate heart rate.

This study is limited to evaluating only the immediate reproducibility of $\mathrm{T}$ wave alternans. Recently, Klingenheben et al. ${ }^{12}$ evaluated the long-term reproducibility of $\mathrm{T}$ wave alternans in patients after myocardial infarction (MI). In this study, 55 consecutive patients who survived a MI underwent a $\mathrm{T}$ wave alternans test $8 \pm 6$ days after MI and after a 1-year follow-up. Of the 55 patients, 32 patients had determinate $\mathrm{T}$ wave alternans test results on both tests, 22 patients had an indeterminate result on one of the two tests, and 1 patient had a MI during the first year of followup and was thus excluded from the analysis. Among the patients with two determinate test re-

Figure 3. T wave alternans tests are shown for the first test (left panel) and the second test (right panel). In each panel, heart rate is displayed on the upper tracing, and $T$ wave alternans is displayed on the lower tracings for the orthogonal vector magnitude lead, and for precordial leads $V_{T} V_{3}$. In this example, there is a long period of alternans in the first test (left panel) in the orthogonal leads and precordial leads $V_{T} V_{3}$ during minutes 13:00-21:00 at heart rates between 105-109 beats/min. This alternans is considered nonsustained, however, because the alternans did not persist when the patient began to exercise and the heart rate increased from 106 to 113 beats/min. The test was interpreted as negative because the final two minutes of the test did not have significant alternans despite heart rates $>110$ beats/min. In the second test (right panel), there is clearly alternans with an onset heart rate of 109 beats/min just below the threshold heart rate of 110 beats/min, and thus, the tracing is classified as positive. Had the onset heart rate been 111 beats/min, the tracing would have been interpreted as negative because alternans that occurs with an onset heart rate $>110$ beats/min is not considered prognostically significant. 

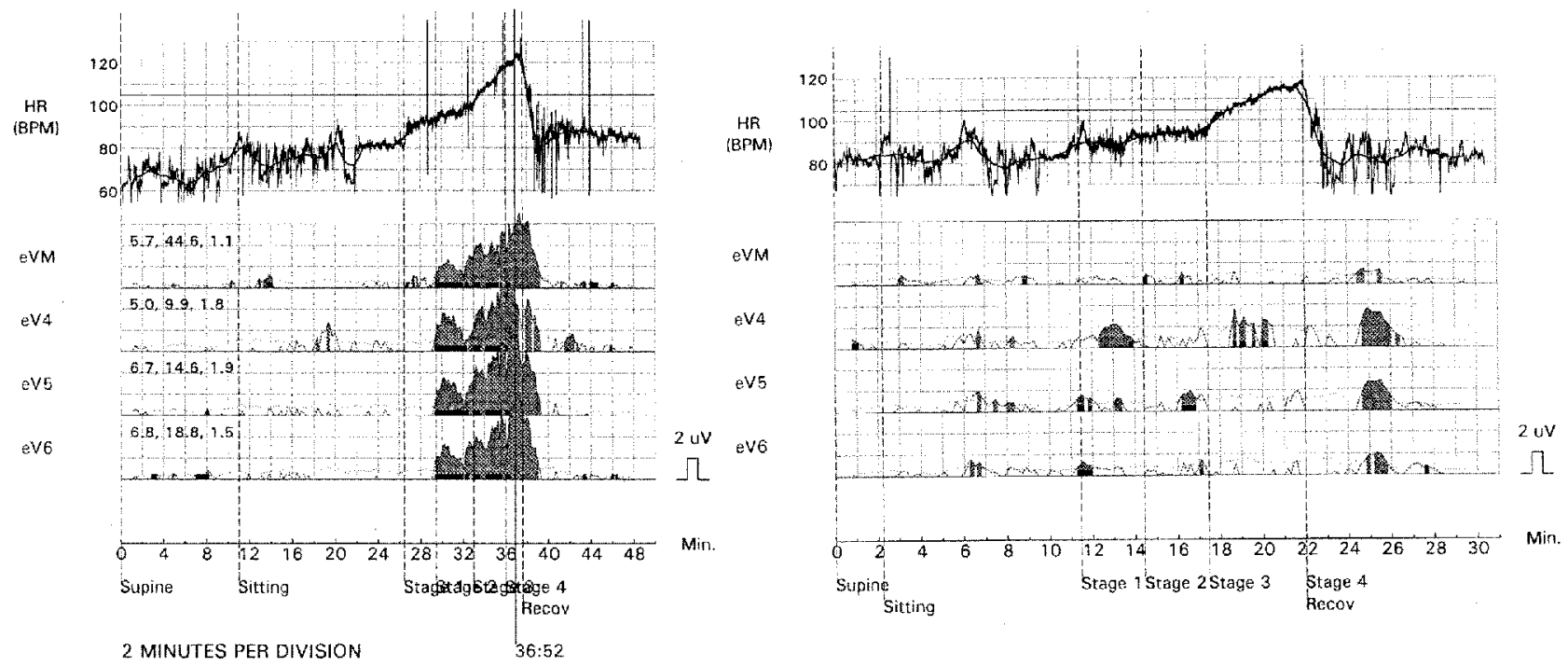

Figure 2. T wave alternans tests are shown for the first test (left panel) and the second test (right panel). In each panel, heart rate is displayed on the upper tracing, and $T$ wave alternans is displayed on the lower tracings for the orthogonal vector magnitude lead, and for precordial leads $V_{4}-V_{G}$ In this example, the first test (left panel) is clearly positive: sustained alternans is present with an onset heart rate of 93 beats/min. The second test (right panel) was negative: there is no sustained alternans and there are two minutes (minutes 20:00-22:00) above a heart rate of 105 beats/min without significant alternans with low noise levels and without ectopic beats.
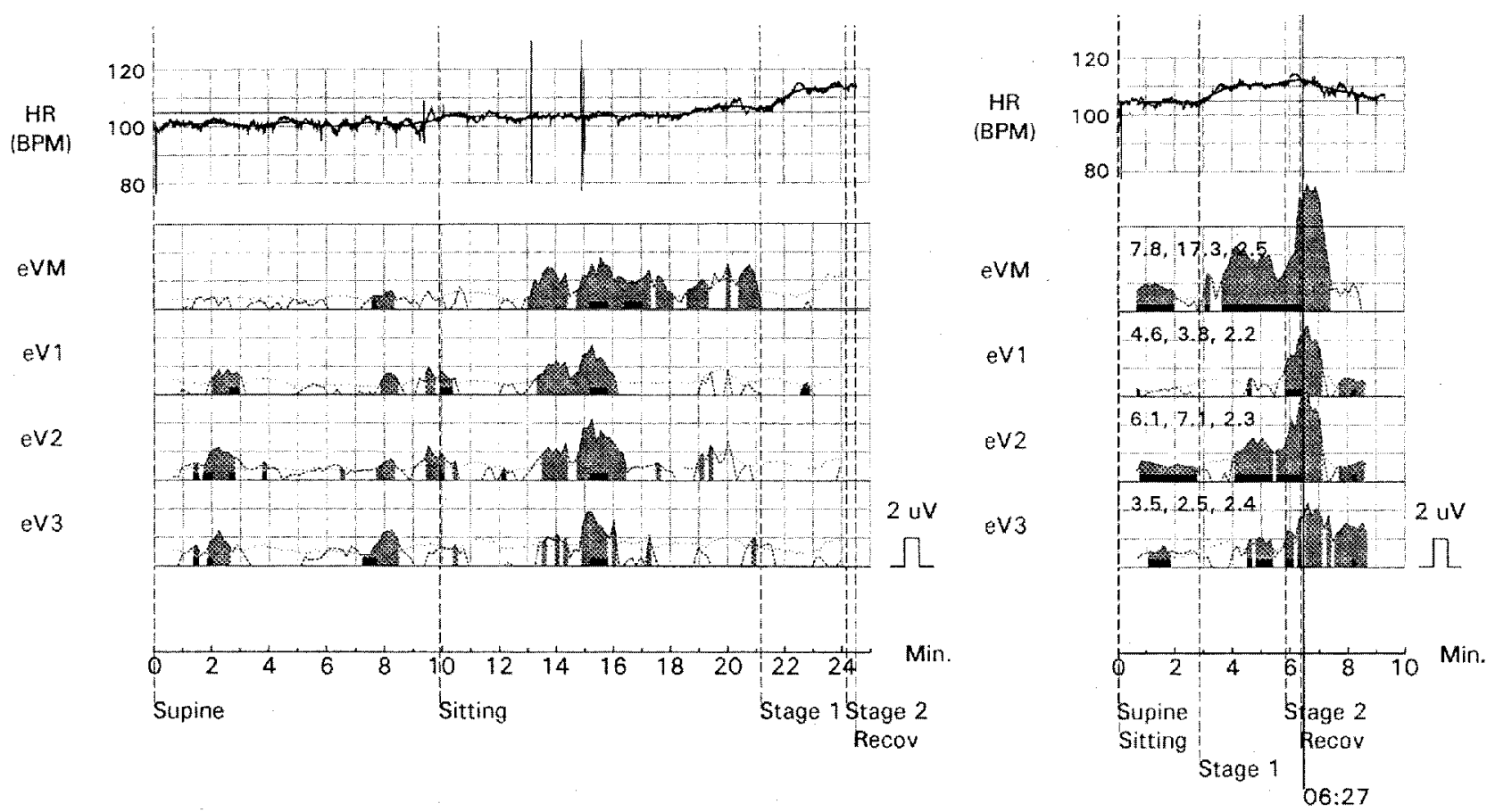

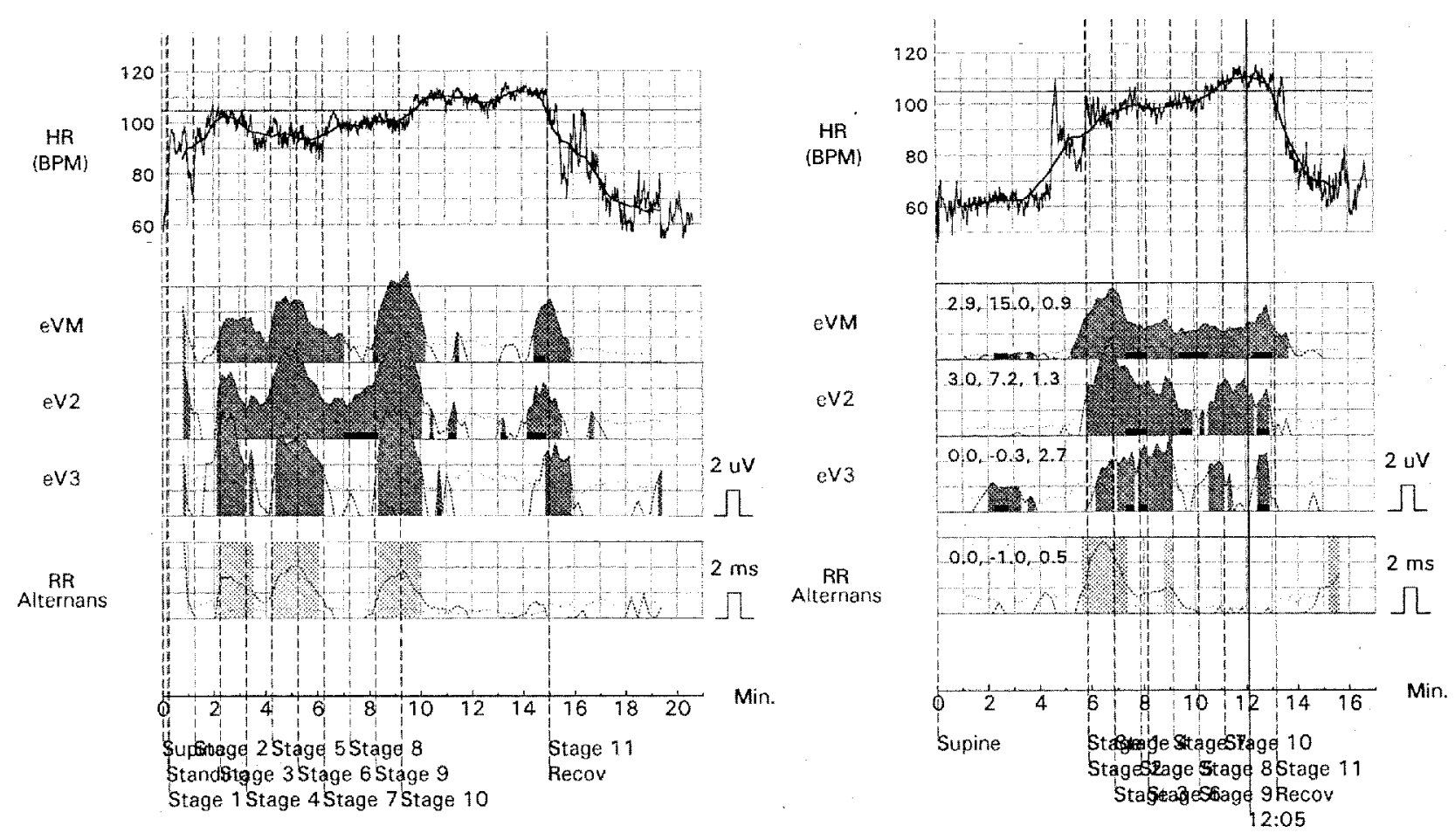

Figure 4. T wave alternans tests are shown for the first test (left panel) and the second test (right panel). In each panel, heart rate is displayed on the upper tracing, and $T$ wave alternans is displayed on the second, third, and fourth tracings for the orthogonal vector magnitude lead, and for precordial leads $V_{2}-V_{3}$. The lowest tracing represents the magnitude of alternans in the $R R$ interval spectrum. When the RR interval alternans exceeds $2 \mathrm{~ms}$ (one small box), the tracing is shaded in grey indicating that RR interval alternans may produce artifactual $T$ wave alternans. In the first test, the initial period of $T$ wave alternans at a heart rate of 90 beats/min at the beginning of the tracing would not be considered significant because it occurs during a period when RR interval alternans is present (see grey shading in the lowest tracing). $T$ wave alternans is not considered significant until later in the tracing when the RR interval alternans has disappeared. This occurs at minute 14:00 following 4 minutes without alternans when the heart rate is 111 beats/min. Because alternans occurring above a heart rate of 110 beats/min is not considered prognostically significant, this tracing is classified as negative. In this patient's second test, $R R$ interval alternans is only present for a brief period (minutes 6:00-7:00) followed by a long period of $T$ wave alternans at and above a heart rate of 90 beats/min that is not confounded by RR interval alternans.

sults, the concordance of the two tests was high $82 \%$. This study provides important additional data supporting the stability and reproducibility of $\mathrm{T}$ wave alternans measured during bicycle exercise.

In an attempt to understand the cause of discordant test results on the two tests, it became clear that some of the discordant test results were related to complexities surrounding the interpretation of $\mathrm{T}$ wave alternans tests. In one patient, the interpretation of one of the two sequential tests was complicated by difficulties interpreting $\mathrm{T}$ wave alternans in the presence of artifacts. In two patients, one of the two sequential tests was extremely difficult to interpret because it was diffi- cult to determine if the alternans was sustained. It is conceivable that in select cases, when the interpretation of one or two tests is difficult, a third test may be useful in gaining confidence in a final interpretation. As we gain a better understanding of how to interpret $\mathrm{T}$ wave alternans tests, it is likely that the interpretation of $\mathrm{T}$ wave alternans tests will become less complicated further enhancing the reproducibility of the test.

One patient, however, demonstrated a clear switch from positive to negative without any obvious explanation. The mechanisms underlying this apparent change in the susceptibility to developing $\mathrm{T}$ wave alternans during exercise need further investigation. 
Table III.

Causes of Indeterminate Test Results

\section{Test 1}

Test 2

$\begin{array}{ll}\text { Indeterminate on both tests } \\ \text { IND (HR) IND (HR) } \\ \text { IND (HR) } & \text { IND (HR) } \\ \text { IND (NSA) } & \text { IND (HR) } \\ \text { IND (HR) } & \text { IND (HR) } \\ \text { IND (HR) } & \text { IND (ectopy) }\end{array}$

Indeterminate on first, determinate on second test

IND (ectopy) POS

IND (NSA, HR) POS

IND (NSA) NEG

IND (NSA) NEG

IND (NSA, ectopy) NEG

Determinate on first, indeterminate on second test

POS

POS

POS

IND (NSA)

IND (NSA)

IND (ectopy)

\section{Conclusions}

T wave alternans measured during bicycle exercise has an acceptable reproducibility when measurements are made from two sequential exercise tests performed within a short period. This data suggests that the measurement of $\mathrm{T}$ wave alternans during exercise is reliable. Repeating the exercise test with a second measure of $\mathrm{T}$ wave alternans during the same session can significantly reduce the proportion of patients with indeterminate test results.

Acknowledgments: The authors thank Beth Mofield who randomly sorted and relabeled all $\mathrm{T}$ wave alternans tracings that enabled the blinded interpretation of the tracings (independent physician readers did not know the sequence of tests or which two tests were from the same patient). They also thank Richard Cohen, M.D., PhD for his assistance as one of the independent physician readers of $\mathrm{T}$ wave alternans tracings.

\section{References}

1. Adam DR, Smith JM, Akselrod S, et al. Fluctuations in T-wave morphology and susceptibility to ventricular fibrillation. J Electrocardiol 1984; 17:209-218.

2. Smith JM, Clancy EA, Valeri CR, et al. Electrical alternans and cardiac electrical instability. Circulation 1988; 77:110-121.

3. Raeder EA, Rosenbaum DS, Bhasin R, et al. Alternating morphology of the QRST complex preceding sudden death. N Engl J Med 1992; 326:271-272.

4. Rosenbaum DS, Albrecht P, Cohen RJ. Predicting sudden cardiac death from $\mathrm{T}$ wave alternans of the surface electrocardiogram: Promise and pitfalls. J Cardiovasc Electrophys 1996; 7:1095-1111.

5. Hohnloser SH, Klingenheben T, Zabel M, et al. T wave alternans during exercise and atrial pacing in humans. J Cardiovasc Electrophysiol 1997; 8:987-993.

6. Gold MR, Bloomfield DM, Anderson KP, et al. A comparison of Twave alternans, signal averaged electrocardiography and programmed ventricular stimulation for arrhythmia risk stratification. J Am Coll Cardiol 2000; 36:2247-2253.

7. Hohnloser S, Cohen RJ. T wave alternans and left ventricular ejection fraction, but not QT variability index, predict appropriate ICD discharge. J Cardiovasc Electrophysiol 1999; 10:626-627.

8. Klingenheben T, Zabel M, D'Agostino RB, et al. Predictive value of $\mathrm{T}$-wave alternans for arrhythmic events in patients with congestive heart failure. Lancet 2000; 356:651-652.

9. Ikeda T, Sakata T, Takami M, et al. Combined assessment of Twave alternans and late potentials used to predict arrhythmic events after myocardial infarction. A prospective study. J Am Coll Cardiol 2000; 35:722-730.

10. Rosenbaum DS, Jackson LE, Smith JM, et al. Electrical alternans and vulnerability to ventricular arrhythmias. N Engl J Med 1994; 330:235-241.

11. Fleiss JL. Statistical Methods for Rates and Proportions. New York, John Wiley \& Sons Inc, 1981.

12. Klingenheben T, Credner SC, Bonsignore M, et al. Long-term reproducibility of analysis of microvolt $\mathrm{T}$ wave alternans after myocardial infarction. (Abstract) PACE 1999; 22:788. 